\title{
Design and Development of the Novel Technology for the Treatment of Patients with the Acute and Chronic Renal Failure
}

\author{
Sakthivel Sankaran, Vishnuvarthanan Govindaraj, M Pallikonda Rajasekaran, Mohamed \\ Mydeen Mohamed Mansoor, Javith Rasha Askar, Abinaya Srinivasan
}

\begin{abstract}
Kidney failure is a condition where the function of kidney gets disabled. In order to sustain in life, dialysis is predominantly adopted. The dialysis is a technical replacement of function of kidney and it is of two types. In considering the long term blood filtering process, the hemodialysis will be an efficient device in replacing the renal functioning but it was currently performed in the stationary mode. In order to fulfill the life supporting requirement, the "Miniaturized portable hemodialysis" device has been introduced which will be portable than the conventional ones. In order to enhance their features, the chambered dialysate technology and the specialized filtering mechanism has been fabricated to this project device. In the process of rectifying the technical errors, sensor indications are implemented for safety measures. In focusing towards the portable mechanism, the battery backup has been applied in this device which can perform patient dialysis in transferable mode.
\end{abstract}

Index Terms: Miniaturization, portable, transportable, sensors, hemodialysis.

\section{INTRODUCTION}

In the total population of the world, 48.7 percent of the people are affected by kidney failure. The kidney failure is the condition where the functioning of the kidney is disabled [1]. The kidney is the pair of bean shaped organs located in the retroperitoneal space. The nephron is the major structural and functional unit of the kidney. The main functions of the kidney are to filter the blood and removal of waste products and electrolyte maintenance of the body. The collected waste products are converted into urine and it is excreted through urine. The kidney failure is of two conditions: acute kidney failure and chronic kidney failure [15] [27]. The acute kidney failure is nothing but the temporary halt of the kidney [2]. This acute level conditioned patients do not need the fistula surgery, for which they will be undergoing a few count of dialysis through the catheter. The chronic level kidney failure

Revised Manuscript Received on December 22, 2018.

Sakthivel Sankaran*, Department of Biomedical Engineering, Kalasalingam Academy of Research and Education, Virudhunagar, Tamilnadu, India. (email: sakthivelsankaran92@ gmail.com)

Vishnuvarthanan govindaraj, Department of Biomedical Engineering, Kalasalingam Academy of Research and Education, Virudhunagar, Tamilnadu, India (email: gvvarthanan@gmail.com)

M Pallikonda Rajasekaran, Department of Electronics and communication Engineering, Kalasalingam Academy of Research and Education, Virudhunagar, Tamilnadu, India. (email: mpraja80@gmail.com).

Mohamed mydeen Mohamed mansoor, Javith rasha Askar and Abinaya Srinivasan, Department of Biomedical Engineering, Kalasalingam Academy of Research and Education, Virudhunagar, Tamilnadu, India. (email: mydeendenim786@gmail.com). is which the functioning of the kidney gets permanently disabled, and this condition will be treated with lifelong dialysis or renal replacement therapy [3]. AV graft or AV fistula will be done for long term medication [19]. The electrolyte imbalance, back pain, leg swelling, the unsedimentation of dead cells, etc are the symptoms of kidney disease [30].

The body cannot function without the support of kidney, and in order to sustain in life, dialysis was adopted [11]. The dialysis is the technical replacement of kidney function which is performed thrice a week. Dialyzer removes the excess water solutes and other toxic products from the blood. This dialysis is performed in a stationary mode and the patient should only reach the hospital to get treated [12]. This hinders the mobility of patients and leads to mental stress. The elder patients experience high inconvenience in frequent travelling to the hospitals [13].

We as a team of biomedical engineers had an intend view on this complication and designed a prototype of miniaturized portable dialysis, which will perform dialysis in conveniently mobile mode and the patients can be performed dialysis in their comfort zone. The function of the device will support the renal failure patients to the fullest.

The flow of the article is constructed in such a way that the concept of complication due to the unavailability of dialysis machine is briefed in section II. It is further preceded with the mechanism of existing technologies in section III. Section IV consists of the proposed solution and Section V says about the system description. In section VI the article gets enclosed with the results and discussion.

\section{QUIETUS RATE}

Kidney failure is a disabled function of the renal elements of the kidney. According to the world health organization report of 2018, the intake supplements of people is the root of the kidney failure [4]. The death rate due to renal failure depend on many factors alike unavailability of machine, technical error, pressure deviation, etc [23]. The medical error also leads to $14 \%$ of death in dialysis [5]. In Kenya, 18 dialysis patients die per day due to the unavailability of machine [29].

The deviation in the pressure acts as the major factor for the elderly patient death [22]. As a result, the morbidity rate due to the renal failure death is about $28 \%$. 


\section{EXISTING SOLUTION}

Artificial kidney or Dialysis is the technical replacement of kidney. The dialysis is the process of eliminating the excess water, waste particles and the toxins of the blood. It also maintains the fluid balance of the body. The dialysis has two methodologies in major: hemodialysis and peritoneal dialysis [6]. The hemodialysis is a process of performing dialysis with dialyser chamber and the ultrafiltration is done in dialyser chamber by taking blood outside the body and electrolyte exchange is also performed [14]. AV fistula surgery and AV graft is done for long term dialysis [21]. The peritoneal dialysis is the process of doing medication in the peritoneal cavity [28]. The abdomen acts as the porous membrane through which the ultrafiltration and electrolyte exchange is done [7]. Once the dialysis process is over, the blood is allowed into vein and the dialysate liquid is drained out. Peritoneal dialysis is done with the fixation of catheter in abdomen area [8]. As considered, efficient hemodialysis is applied into the various applications like Nocturnal dialysis, which is performed at night during sleeping and it wants around 7 hours of time [9] [10]. The NX Stage is a company that currently provides the homely hemodialysis, which comforts the patient by rendering dialysis at home [18] [24]. Continuous renal replacement therapy is accident mode dialysis and also long term dialysis is performed around 24 hours. The multimedications can be done along with dialysis in general [16].

\section{PROPOSED SOLUTION}

Our team have undergone the depth literature survey and hospital survey and analyzed the barriers of renal failure patients.

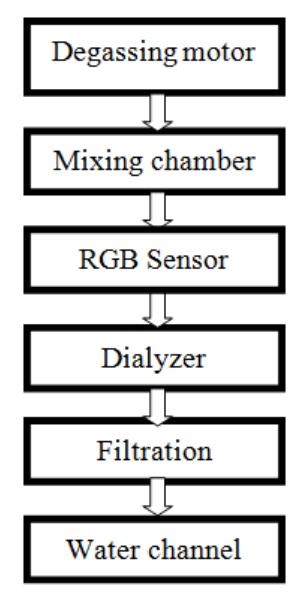

Fig. 1.Methodology

The renal disabled patients are highly suffering with their non-mobility medication process. Our team has viewed it as a serious issue and we decided to bring a solution for it, there we came up with an idea "Miniaturized Portable Hemodialysis".

The project device (Fig. 1) which we have designed will be solidly supporting the renal failure patients with both the ultra-filtration process and electrolyte exchange process. Initially, the water and the bicarbonate solution get suctioned by the degassing motors and allowed into the mixing chamber. The mixing chamber will mix both the water and bicarbonate solution and the dialysate solution is prepared in this chamber. The dialysate solution is transported to the dialyzer membrane, where the ultra-filtration and the electrolyte exchange are done.

During the dialysis process, the used dialysis is eliminated through the exit tube, which is connected to the charcoal electrolyte filter. The charcoal electrolyte filter will filter the used dialysate solution and reduce the bicarbonate content in the solution and the remaining water content can be used as a water supplement for the dialysis process [20]. The RGB sensor is fixed in the exit tube to monitor the blood leakage from the dialyzer membrane [17]. The process is kept on repeating as a cycle and the medication is done.

\section{COMPARITIVE ANALYSIS}

The evolution of dialysis machine has occurred based on the essentiality and need of the patients. The mechanical construction of hemodialysis has also altered with the convenience for installment of updated technologies. In comparing the current marketable dialysis machine and proposed device, the water recycling process hasn't been enhanced till now. [20]The current dialysis machine requires a separable water plant for the supply of adequate water needed for medication [2]. Whereas, in our project device the electrolyte charcoal filter has been fixed to reduce the need of water. The motors which are used in current marketable device are over capacitive for the process of dialysis so we have constructed the device with the mini and low volt motors. [11]The priming system is very essential in case of long tubing like normal hemodialysis machine, but we have made it with convenient length which is easy cleanable and affordable. [17]The steps towards physician less dialysis is very much less in the current mechanism, but the project device is well enhanced with the safety measures using sensor alert systems. By increasing rate of capillary gravitational pressure in the machine with cycled liquid flow in our project device [29], the role play if blood pump becomes unneeded. In order to make the device as the easy portable one some of the inbuilt gadgets have been miniaturized with microcontrollers.

\section{SYSTEM SOLUTION}

The miniaturized portable hemodialysis is a project device which is designed to be used as a portable medication device (Fig. 2). Initially, the water and the dialysate solution get suctioned by the degassing motors 1 and 2, which works based on the operational inputs from the power supply of single $12 \mathrm{~V}(1.3 \mathrm{~A})$ battery and the speed of the motor is controlled by the Pulse With Modulation (PWM). Both the solutions are allowed to the mixer chamber where both the solutions get mixed in the ratio of $34: 1$, and the dialysate solution is prepared as an outcome of this.

The dialysate solution is transported to the dialyser membrane by degassing motor 3 , which is controlled by the PWM for controlling it in specific speed. The dialyser membrane is a chamber where ultra-filtration and electrolyte exchange greatly occurs. During the dialysis process, the used dialysis is eliminated through the exit tube, the exit tube is get connected to the stainless steel charcoal electrolyte filter.

The stainless steel charcoal electrolyte filter will filter the used dialysate solution and 
reduce the bicarbonate content in the solution up to $55 \%$ and the remaining water content can be used as a water supplement for that dialysis process. By this, filtering process the water content used for a dialysis, and is reduced into half. It will be convenient for portable purpose. The RGB sensor is fixed in the exit tube to monitor the blood leakage from the dialyzer membrane. The RGB sensor receives its control from the Arduino UNO R3. The detection of blood program is dumped into the sensor through Arduino. If the sensor detects the red colour, the buzzer connected to Arduino starts the alarm.

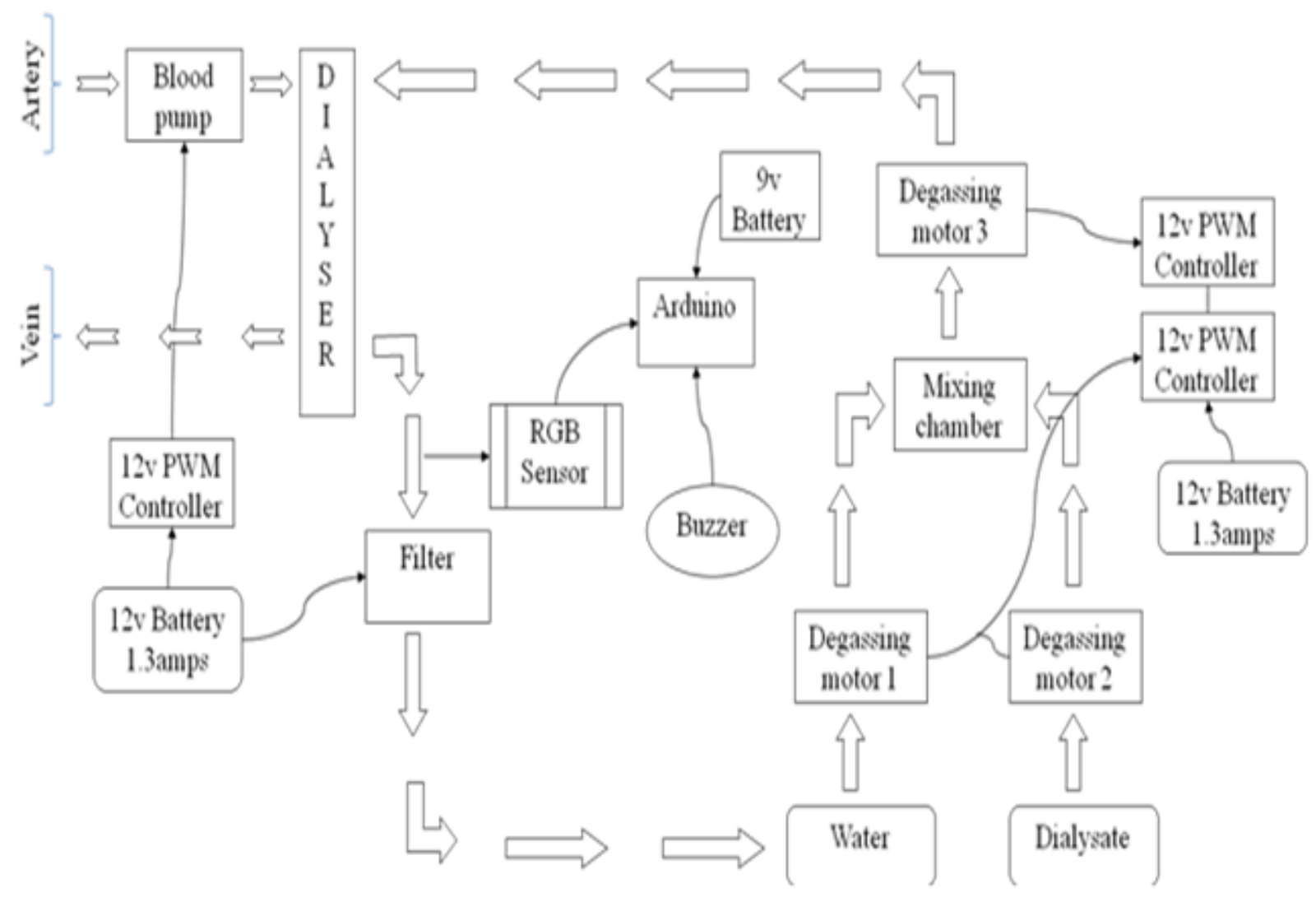

Fig. 2.Block diagram of dialysis machine

\section{A. Mixing Chamber}

The mixing chamber is used to prepare the dialysate solution by combining water and sodium bicarbonate. The water and the sodium bicarbonate solution (NaHCO3) are allowed inside the mixing chamber (Fig. 3), which gets mixed with the mixing coupler by the rotating action of $5 \mathrm{v}$ motor. The motor functions with the power supply from the $9 \mathrm{~V}$ battery. This setup is enclosed with the plastic lids.

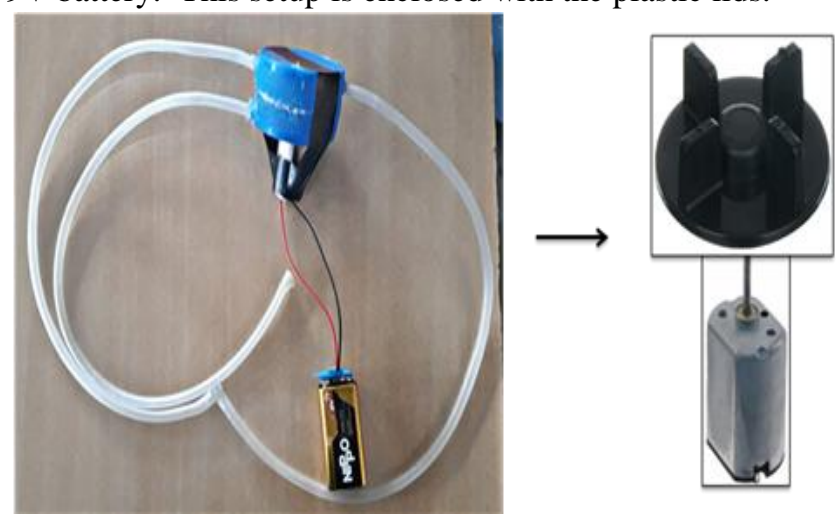

Fig. 3.Mixing chamber

\section{B. Dialyzer}

Dialyzer is a membrane and tends to be the main and major component in hemodialysis that performs the function of ultra filtration and electrolyte exchange. The Fresenius Helixone FX 5 dialyzer (Fig. 4) with the dual dialysate flow connection and dual blood flow connection is specially used for the high flux dialysis for the patients who are having normal pressure level. The total membrane pressure of the dialyzer is up to $600 \mathrm{mmhg}$. It is the dual sterile porous membrane. The dialyzer is a porous membrane which performs the ultra filtration through the porous alignment process. The electrolyte supplement residuals of blood will be removed through dialyzer.

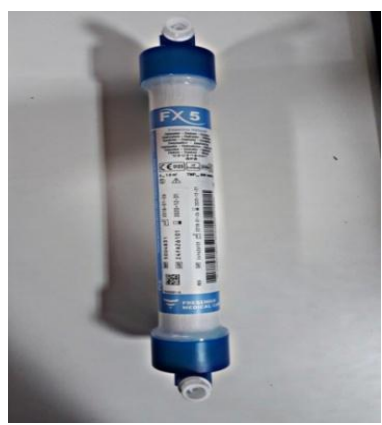

Fig. 4.Dialyzer

\section{Degassing Motor}

The degassing motor consists of two connections, where one drives/draws the liquid from I/P port and delivers the same through O/P port. This is a direct current motor (Fig. 5) and the voltage level is $12 \mathrm{~V}$ and the rated current is in the rage of 500ma. The water flow rate is limited up to the $100 \mathrm{ml} / \mathrm{min}$. The pressure range

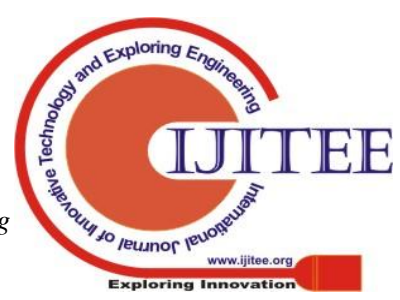


of the water is up to 14.5 PSI. The noise level of the motor is up to $65 \mathrm{~dB}$. The motor performance will get deviated according to its source of power. The speed of motor is adjusted with the PWM. The $12 \mathrm{~V}(1.3 \mathrm{~mA})$ battery can be used as a power source for functioning this motor.

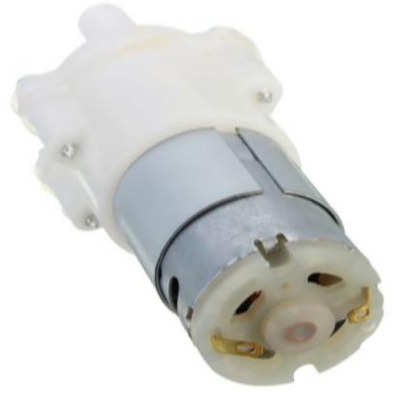

Fig. 5.Degassing motor

\section{Filter}

The filter will separate the non-soluble precipitate like sodium bicarbonate in a corresponding ratio which filters in the rate of $100 \mathrm{ml} / \mathrm{sec}$. The stainless steel charcoal electrolyte (typically combined in one step) is fixed in the inner chamber of filter (Fig. 6). It separates the bicarbonate contents in solution. The existing rate of filter is 10 months. In the case of expiry, it will lose its efficiency. The ratio of calcium and magnesium was filtered up to $55 \%$ in the bicarbonate solution.

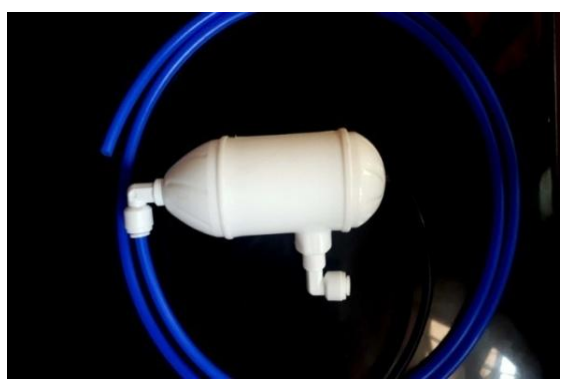

Fig. 6.Charcoal Electrolyte Filter

\section{E. PWM Controller}

The pulse with modulation control board (Fig. 7) is used to control the rotation per minute of the motor. The rotating module of the motor is get controlled by the PWM with the varied pulse provided by the controller the positive and negative module of both the motor and the battery is connected to the PWM, and it controls the speed of motor which will reduce the suction and supply capacity of the motor and the quantity of solution that can be driven.

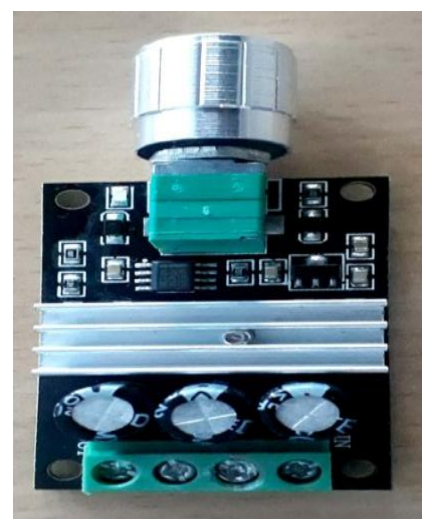

Fig. 7.Pulse with modulation Controller

\section{F. RGB Sensor}

RGB sensor or color senor is used to convert the color light to frequency. This color sensor module is combined with silicone photodiode and frequency converter. This color sensor (Fig. 8) allows both the digital input and digital output. The output frequency is differentiated in three scales. This specific module is easy to connect with the breadboard. The power supply is in range of 2.7 volt to 5.5 volt. Color sensor module is highly used in medical imaging equipments. The color coding is user friendly and so the program can be easily implemented.

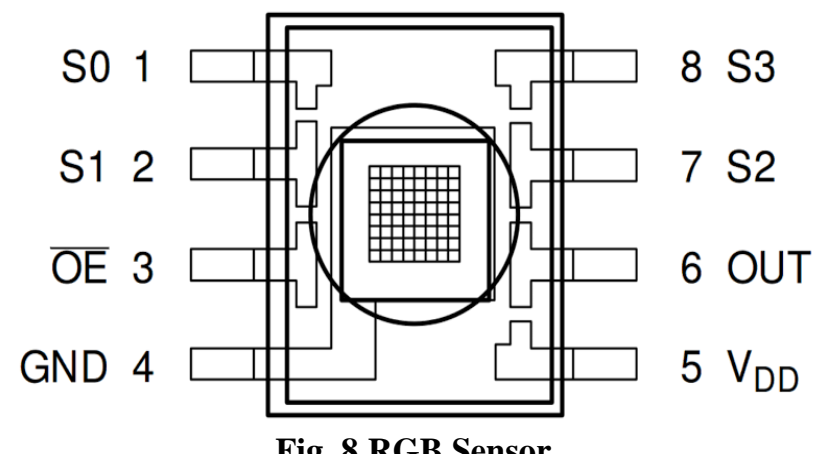

\section{G. Arduino}

Arduino UNO R3 (Fig. 9) is a sensor which is the combination of hardware and software. It is the microcontroller design layout which is fabricated based on the microchip. It is made in a single board and acts as a communicative object between digital devices. The arduino is employed in this device, and this is used to control the RGB sensor by code and it is also used to alarm the buzzer if the sensor detects red color.

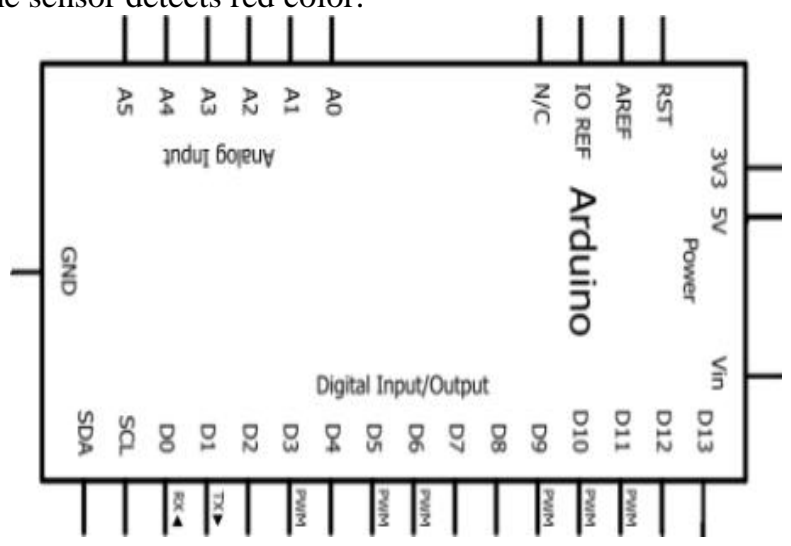

Fig. 9.Arduino

\section{H. Acidic Sodium Bicarbonate}

The sodium bicarbonate solution is prepared with the combination of RO water and bicarbonate salt (Fig. 10). The combination ratio of sodium bicarbonate solution is 1(NaHCO3): 34(RO Water). In 1 liter of water, 60 grams of sodium bicarbonate salt is added to prepare a bicarbonate solution. This solution acts as a major component of dialysate solution. The acidic solution is added with the NaHCO3 to make it into acidic form. 


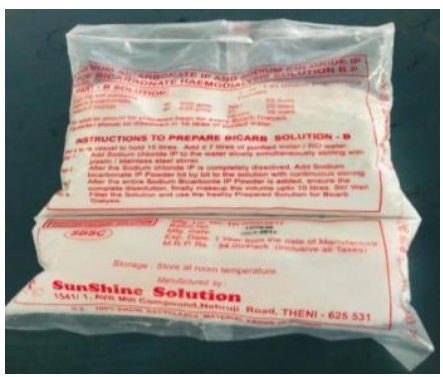

Fig. 10. Acidic Sodium Bicarbonate

\section{RESULT AND DISCUSSION}

As the conclusion, the portable dialysis mechanism will surely make the patient life better. The complete functioning prototype performs the actual function of hemodialysis in the portable structure. The front side (Fig. 11) of prototype consists of the source chamber, from which the water and bicarbonate solution gets suctioned, and the mixing chamber is also localized. The back side (Fig. 12) of the prototype setup consists of dialyzer membrane, sensor segment and the filter chamber. The total setup weighs up to 4.5 kilogram.

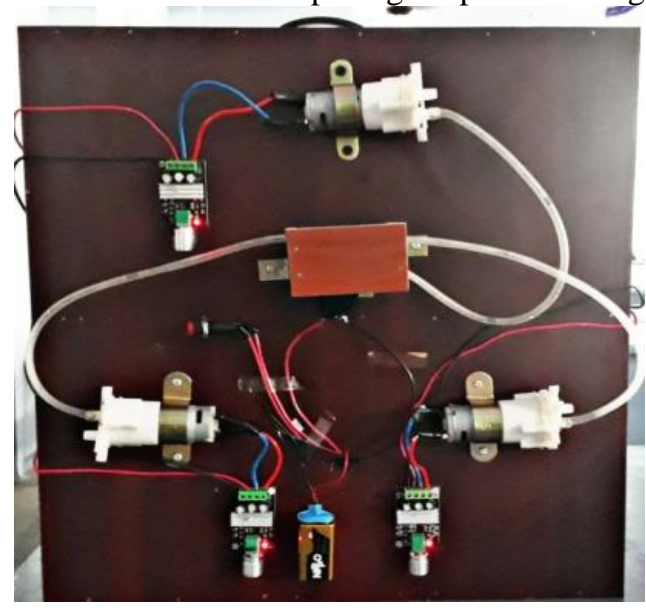

Fig. 11. Front Panel of the indigenous prototype

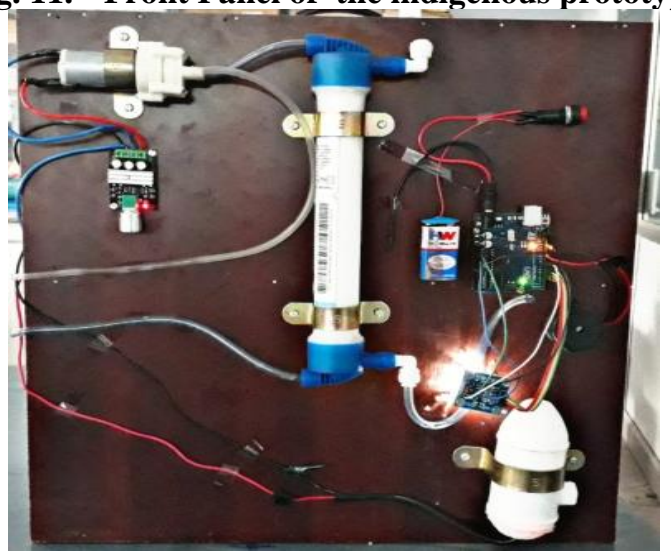

This project device will be highly helping the renal failure patients in handling their medication at their convenience zone, which will apparently give much more relief to the patients and can improve their health conditions.

\section{CONCLUSION}

In this paper, we have discussed about the dialysis machine which is developed for the betterment of the affected individuals. The portable hemodialysis machine has been enhanced lot after a depth literature survey, repetitive troubleshooting and analyzing an experienced evolution
Fig. 12. Back panel of the indigenous prototype

which to satisfy the portable necessity. The device liquid flow and rate of ultra filtration is constructed in such a way of maintaining dialytic weight of the patients and balancing the long term medication process. The portable hemodialysis machine will constantly support the patients to undergo their dialysis in their comfort zone.

\section{ACKNOWLEDGEMENT}

The authors would like to thank the management of Kalasalingam Academy of Research and Education. The authors also extend their regards to the Department of Biomedical Engineering for their support. The authors deliver their sincere thanks to KLU NI-Technology Innovation Centre for providing computational and DC laboratory of Electrical and Electronics Engineering Department for providing the hardware testing facilities.

\section{REFERENCES}

1. M Olivier J. Wouters et. al., Early chronic kidney disease: diagnosis, management and models of care Doi: 10.1038/nrneph.2015.85.

2. Simonetta genovesi et. al., Sudden death in end stage renal disease Comparing hemodialysis verus peritoneal dialysis.

3. Rajnish mehrota et. al., The current state of peritoneal dialysis 2016 Doi: 10.1681/ASN.2016010112.

4. Sean M.bagshaw et. al., Precision continuous renal replacement therapy and solute control Doi:10.1159/00448507.

5. Bhanu Prasad et. al., Early mortality on continuous renal replacement therapy (CRRT): the prairie CRRT study Doi: 10.1186/s40697-016-0124-7.

6. Rajnish mehrota et. al., The current state of peritoneal dialysis Doi: 10.1681/ASN.2016010112.

7. Michael j. Ficher et. al., Predialysis nephrology care and dialysis related health outcomes among older adult initiating dialysis Doi: 10.1186/s12882-016-0324-5.

8. Thiago gomes romano et. al., barufi Insights about serum sodium behaviour after 24 hours of continuous renal replacements therapy Doi: $10.5935 / 0103-507 x .20160026$

9. Xu Deng,Yifan Xie \&amp;Aihua Zhang Advance of autophagy in chronic kidney diseases Doi: 10.1080/0886022X.2016.1274662.

10. Silvia De Rosa et. al., Management of chronic kidney disease patients in intensive care unit: Mixing acute and chronic illness 2017 Doi:10.115/000452650

11. Sabitha vadakedath and Venkataramana kandi cureus Dialysis: A Review of the Mechanisms Underlying Complications in the Management of Chronic Renal Failure 2017 Doi $10.7759 /$ cureus. 1603 .

12. Ali momeni et. al., Comparison of complications of arteriovenous fistula with permanent catheter in hemodialysis patients: A six month follow up 2017 Doi: 10.4103/2277-9175.213666.

13. Marion Morena et. al., Treatment tolerance and patient reported outcomes favour online hemodiafiltration compared to high flux hemodialysis in elderly Doi: 10.1016/j.kint.2017.01.013.

14. Rajeev A. Annigeri et. al., Renal support for acute kidney engineering in the developing world Doi:10.1016/j.ekir.2017.04.006

15. Peter K. Moore et. al., Management of acute kidney injury: Core curriculum 2018 Doi: 10.1053/j.ajkd.2017.11.021.

16. Raymond Vanholder et. al., Deleting Death and Dialysis: Conservative Care of Cardio-Vascular Risk and Kidney Function Loss in Chronic Kidney Disease (CKD) 2018 Doi 10.3390/tonins 10060237 .

17. Sara alijassmi et. al., Smart portable kidney dialysis control and monitoring system 2018 Doi: 10.13140/RG.2.2.13421.69604.

18. Sabrina Haroon \&Andrew Davenport Pages Hemodialysis at home: review of current dialysis machines Doi: 10.1080/17434440.2018.1465817.

19. Wolfgang bieser and Markus welsch Effectiveness of a New Single-Needle Single-Pump Dialysis System with Simultaneous Monitoring of Dialysis Dose Doi: 10.1111/aor.13149.

20. Gunjeet Kaur1 et. al., Cost of hemodialysis in a public sector 
tertiary hospital of India Doi: $10.1093 / \mathrm{ckj} / \mathrm{sfx} 152$

21. Mohamed ali shafiee et. al., The impact of hemodialysis frequency and duration on blood pressure management and quality of life in end-stage renal disease patient Doi: 10.390/Healthcare5030052.

22. Rachael C Walk et. al., Home hemodialysis: a comprehensive review of patient-centered and economic considerations Doi: 10.2215/CJN.05501008

23. Manish Saha and Michael Allon Diagnosis, Treatment, and Prevention of Hemodialysis Emergencies Doi: 10.2215/CJN.05260516.

24. Sabitha vadakedath and Venkataramana kandi cureus Dialysis: A Review of the Mechanisms Underlying Complications in the Management of Chronic Renal Failure Doi: 10.7759/cureus.1603.

25. Qingyun zhu et. al, Clinical evolution of continuous renal replacement therapy in combination with ultrasound guided percutaneous transhepatic gallbladder drainage for acute severe pancreatitis: a retrospective study Doi:10.1159/000485437.

26. Soren Christiansen, Steffen Christiansen Timing of renal replacement therapy and longterm risk of chronic kidney disease and death in intensive care patients with acute kidney injury Doi: 10.1186/s13054-017-1903-y.

27. Jihane J. Hajj and Krzystof lauda Home hemodialysis treatment as an effective yet utilized treatment modality in the United States Doi: 10.3390/healthcare5040090.

28. Graham Woodrow, Stanley L. Fan, Renal Association Clinical Practice Guideline on peritoneal dialysis in adults and children Doi: 10.1186/s12882-017-0687-2.

29. Bazae V et. al In vitro trials of a wearable artificial kidney (WAK) Doi:10.5301/ijao.5000651.

30. Gura $\mathrm{V}$ et al., A wearable artificial kidney for patients with end-stage renal disease PMCID: pmc4936831.

\section{AUTHORS PROFILE}

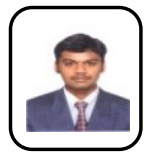

Sakthivel Sankaran received B-Eng. in Electronics and Communication Engineering, May 2013 and M.Tech in (Biomedical Engineering) at VIT University, Vellore, May 2015. Currently working as an Assistant Professor in Biomedical Engineering department at Kalasalingam Academy of Research and Education, Krishnankoil. His research interests are Biomechanics, Rehabilitation Engineering and Biomedical Instrumentation. He has so far published more than 09 papers in national and international journals and conferences. He is a Fellow of Indian Society For Technical Education (ISTE), Institute of Electrical and Electronics Engineers (IEEE), Biomedical Engineering Society of India (BMESI).

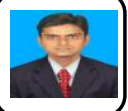

Dr.G.Vishnuvarthanan, born in 1986, has research stints in the avenues of medical image processing and artificial intelligence. He was awarded $\mathrm{PhD}$ in the year 2015 and bachelor's degree in Instrumentation and Control Engineering by 2007, and Master's Degree in VLSI by 2009. He has more than ten years of teaching and research experience and has his affiliation as Associate Professor with the Department of Biomedical Engineering of School of Bio and Chemical Sciences in the Kalasalingam Academy of Research and Education, Tamilnadu, India.

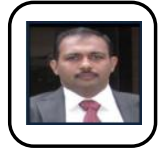

Pallikonda Rajasekaran Murugan, Born in Srivilliputhur, Virudhunagar District of TamilNadu in 1980, he had his schooling in the same town and graduated in Electronics and Instrumentation Engineering in 2001 from Shanmugha College of Engineering, Thanjavur and completed his M.Tech. degree in 2002 with second Rank in SASTRA University. He pursued his doctoral programme in Anna University, Chennai. Starting as a Lecturer in 2003, he became Asst. Professor in 2008, Associate Professor in 2009 and Professor in 2012 in Kalasalingam Academy of Research and Education. He had a deep involvement in Bio-signal Processing research. His work on the Image Segmentation for identification of brain tumour and image reconstruction and compression using medical images for diagnosis. Over 150 B.Tech students, 75 M.Tech students, and 8 Doctorates stand testimony for his productivity in Image Processing, Wireless Sensor Networks, and Biomedical Instrumentation research. He has so far published more than 50 papers in national and international journals and conferences. He is a Fellow of Indian Society For Technical Education (ISTE), Institute of Electrical and Electronics Engineers (IEEE), Asia-Pacific Chemical, Biological\&amp; Environmental Engineering Society (APCBEES), Institution of Engineers (India)(IE), International Association of Engineers (IAENG) and International Association of Computer Science and Information Technology (IACSIT).

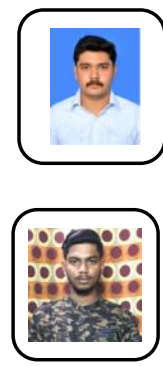

Mohamed mydeen Mohamed mansoor studied his B.Tech Biomedical Engineering at Kalasalingam Academy of Research and Education, Krishnankoil.

Javith rasha Askar studied his B.Tech Biomedica Engineering at Kalasalingam Academy of Research and Education, Krishnankoil.

Abinaya Srinivasan studied her B.Tech Biomedical Engineering at Kalasalingam Academy of Research and Education, Krishnankoil. 